\title{
Fusão de Face e Forma de Andar para Reconhecimento Biométrico: Revisão Sistemática de Literatura
}

\author{
Alternative Title: Fusion of Face and Gait for Biometric Recognition: Systematic \\ Literature Review
}

\author{
Edenilton L. Oliveira \\ Universidade de São Paulo \\ Av. Arlindo Bettio, 1000 \\ 03828-000 São Paulo, SP, \\ Brasil \\ edenilton@usp.br
}

\author{
Clodoaldo A. M. Lima \\ Universidade de São Paulo \\ Av. Arlindo Bettio, 1000 \\ 03828-000 São Paulo, SP, \\ Brasil \\ c.lima@usp.br
}

\author{
Sarajane M. Peres \\ Universidade de São Paulo \\ Av. Arlindo Bettio, 1000 \\ 03828-000 São Paulo, SP, \\ Brasil \\ sarajane@usp.br
}

\begin{abstract}
RESUMO
Neste artigo é apresentada uma revisão sistemática de literatura (RS) conduzida no domínio da biometria multimodal, considerando a fusão das características biométricas face e forma de andar. Sistemas biométricos baseados em face e forma de andar são úteis em ambientes não controlados com a presença de usuários não cooperativos. Esta RS inclui 18 estudos primários cuja análise permitiu concluir que embora o tema em questão já apresente algumas tendências, há ainda lacunas importantes que precisam ser investigadas.
\end{abstract}

\section{Palavras-Chave}

Sistema Biométrico Multimodal, Fusão, Forma de Andar, Face, Revisão Sistemática.

\begin{abstract}
In this paper is presented a systematic literature review (SLR) conducted in the field of multimodal biometrics, considering the fusion of biometric characteristics of face and gait. Biometric systems based on fusion of face and gait are useful in non-controlled environments with non-cooperative users. This SLR includes 18 primary studies which allowed the conclusion that although the theme presents some trends, there are still important gaps that need to be investigated.
\end{abstract}

\section{Categories and Subject Descriptors}

H.4.m [Information Systems Applications]: Miscellaneous; I.5.4 [Pattern Recognition]: Applications-Computer Vision

\section{General Terms}

Documentation

Permission to make digital or hard copies of all or part of this work for personal or classroom use is granted without fee provided that copies are not made or distributed for profit or commercial advantage and that copies bear this notice and the full citation on the first page. To copy otherwise, to republish, to post on servers or to redistribute to lists, requires prior specific permission and/or a fee.

SBSI 2016, May 17th-20th, 2016, Florianópolis, Santa Catarina, Brazil Copyright SBC 2016.

\section{Keywords}

Multimodal Biometric System, Fusion, Gait, Face, Systematic Review

\section{INTRODUÇÃO}

Sistemas Biométricos Multimodais integram dois ou mais sistemas biométricos, com a motivação de fornecer uma solução mais confiável para o problema de identificação de pessoas [6]. Diferentes características biométricas e estratégias de integração podem ser usadas para construir um sistema biométrico multimodal. Por exemplo, as características biométricas face e forma de andar podem ser integradas para atender os requisitos de um sistema biométrico implantado em um ambiente não controlado, suscetível a situações adversas como alterações de ângulo e distância de visualização de uma câmera de vídeo, ou mudanças de iluminação, e no qual os usuários não necessariamente concordam em fornecer suas amostras biométricas. Uma estratégia para implementar a integração das características biométricas é a "integração" baseada no nível de fusão", impondo ao sistema um processamento paralelo e mais rápido. A biometria com tais características é útil, por exemplo, em sistemas de vigilância para identificação da presença de indivíduos que representam ameaças em ambientes como aeroportos, praças, escolas, etc. Outros exemplos de sistemas biométricos multimodais e suas aplicações são apresentados em [10] [5].

Esta revisão sistemática de literatura (RS) identifica, avalia e interpreta os estudos primários relevantes no domínio da biometria multimodal que integra as características biométricas face e forma de andar usando integração no nível de fusão. A motivação para apresentação desta RS é a ausência de estudos secundários sistemáticos abordando este tema. A partir das buscas realizadas na condução desta RS foi possível encontrar apenas um estudo secundário livre, não sistemático [8], no qual os autores apresentam uma visão geral das principais técnicas usadas em sistemas de reconhecimento biométrico pela fusão de face e forma de andar, restringindo os estudos primários a apenas aqueles que usam a abordagem livre de modelo - uma restrição que não foi aplicada na presente RS. Este artigo é organizado como segue: a seção 2 apresenta os principais conceitos teóricos relacionados ao tema da RS; a seção 3 descreve o método utilizado nessa RS; a seção 4 apresenta os resultados e 
discussões frente as questões de pesquisa estabelecidas; a seção 5 apresenta uma análise de possíveis ameaças à validade da RS, e por fim, a seção 6 apresenta as conclusões.

\section{FUNDAMENTAÇÃO TEÓRICA}

Um sistema biométrico é um sistema de reconhecimento de padrões que tipicamente: recebe como entrada uma instância biométrica coletada do indivíduo por meio de sensores, extrai um vetor de características relevantes, o compara com as características do indivíduo previamente salvas em um banco de dados, e fornece a identidade do indivíduo como saída [14]. Tais sistemas operam em modo de verificação ou identificação. No modo de verificação, um indivíduo que deseja ser reconhecido alega uma identidade, geralmente por meio de um número de identificação, o sistema capta sua característica biométrica, procura o padrão biométrico do indivíduo no banco de dados e o compara com as entradas respondendo se a pessoa é ou não quem ela alega ser. Já no modo modo de identificação, o indivíduo é reconhecido de acordo com a identidade de maior verosimilhança com a instância biométrica de entrada, encontrada em uma busca por todo o banco de dados do sistema.

Um sistema biométrico clássico possui quatro módulos: sensorial, extração de características, classificação (ou mapeamento) e decisão [19]. Esses módulos são responsáveis respectivamente por: fazer a leitura das amostras biométricas; extrair as características relevantes das amostras e criar vetores de características que as represente; mapear cada vetor de características para uma variável independente comumente chamada de escore; aplicar uma função (geralmente uma função sinal) capaz de tomar a decisão final do sistema com base no valor da variável escore.

Um sistema biométrico multimodal integra dois ou mais sistemas biométricos. A forma de integração pode ser baseada na arquitetura ou baseada no nível de fusão (BNF). Na primeira forma, o processamento de cada características biométrica ocorre em cascata podendo ser de forma serial ou hierárquica [14]. Para a integração BNF, principal interesse nesta RS, o processamento das características biométricas é em paralelo e existem quatro níveis de fusão possíveis: nível sensorial, nível de extração de características, nível de escores, e nível de decisão. Os dois primeiros são ditos níveis de pré-classificação pois são executados antes do módulo de classificação, e os dois últimos são ditos níveis de pós-classificação pois ocorrem depois do módulo de classificação. Para um detalhamento sobre o que caracteriza cada um desses níveis de fusão, veja [19].

Um sistema biométrico multimodal baseado na fusão de face e forma de andar pode variar de acordo com: a forma de aquisição dos dados (cooperativa - com supervisão humana; não cooperativa - sem supervisão humana); o tipo de ambiente (controlado - se houver controle sobre variáveis como ângulo e distância do sensor, ou iluminação; ou não controlado); e forma de integração (baseada na arquitetura ou baseada no nível de fusão). Em geral, o ambiente controlado é interno (indoor) e o ambiente não controlado é externo (outdoor). Neste último caso, a aquisição sofre muitos tipos de variações: influência da luz solar, efeitos de sombra, complexidade de fundo, etc. Na prática não existem bancos de dados que representem totalmente as condições de ambientes não controlados.

O reconhecimento biométrico com fusão de face e forma de andar podem ser de abordagem livre de modelo (ou baseada em aparência) ou abordagem baseada em modelo [2]. Na primeira, as características são extraídas das imagens da face ou das sequências de imagens de silhuetas da forma de andar. Já na segunda, a face ou o corpo do indivíduo é reconstruído usando um modelo geométrico a partir do qual são extraídos as características.

\section{MÉTODO}

Uma RS é uma forma de identificar, avaliar e interpretar todos os trabalhos disponíveis para uma determinada questão de pesquisa, tópico de uma área ou fenômeno de interesse, caracterizando-a como um estudo secundário [16]. A diferença entre uma RS e os demais tipos de estudos secundários é a sistematização com a qual ela é conduzida. Essa sistemática garante que a mesma seja científica, replicável e livre de viés [16]. A presente RS segue as diretrizes propostas em [16], que especifica três passos: (i) planejamento (seções $3.1 ; 3.2 ; 3.3$ ); (ii) condução (seção 3.4) e (iii) relatório (seção 4).

\subsection{Justificativa para a Revisão Sistemática}

Não foi possível encontrar nas principais bases de dados de trabalhos científicos nenhuma RS abordando o tema aqui estudado. Os estudos primários avaliados na presente RS foram desenvolvidos por pesquisadores de diferentes grupos de pesquisa interessados na fusão de face e forma de andar para o reconhecimento biométrico, portanto, faz-se útil avaliar o que está sendo desenvolvido nessa área num contexto panorâmico para então compartilhar com outros potenciais pesquisadores interessados; desta forma, outros grupos de pesquisa podem começar seus trabalhos a partir dos resultados dessa RS economizando tempo e minimizando a probabilidade de não acessar um estudo primário relevante.

\subsection{Questões de pesquisa}

Assumindo a necessidade para uma RS, as questões de pesquisa foram elaboradas com base em [16]. Considerando um conjunto de configurações em um sistema biométrico multimodal com fusão de face e forma de andar que inclui: (i) as abordagens livre de modelo e baseada em modelo; (ii) o ambiente das cenas interno, externo e heterogêneo; (iii) o esquema de integração BNF Nível Sensorial, BNF Nível de Extração de Características, BNF Nível de Escores, BNF Nível de Decisão e outros; (iv) as condições de ambiente controlado e não controlado; as questões de pequisa são:

Q1. Quais são as configurações mais exploradas? Q1.1. Quanto à abordagem? Q1.2. Quanto aos ambientes das cenas? Q1.3. Quanto ao esquema de integração? Q1.4. Quanto às variações de ambiente?

A Q1 é uma questão genérica que foi decomposta em quatro questões mais específicas. Cada uma das questões mais específicas foi utilizada para motivar a verificação de tendências das pesquisas em cada quesito. Um destaque deve ser feito para a questão Q1.4, que foi utilizada para avaliar quais fatores causadores de variações intraclasses estão presentes nos experimentos apresentados nos estudos e que portanto, caracterizam a complexidade com a qual os sistema biométrico com fusão de face e forma de andar pode ter que lidar. Essa complexidade pode ser caracterizada, por exemplo, por alterações de ângulos de aquisição de dados, características das roupas dos indivíduos, tempo decorrido entre as amostragens e condição de transporte (de mochilas ou bolsas, por 
exemplo). A resposta a essa questão pode direcionar novos pesquisadores para questões ainda em aberto no tema.

Q2. O método é invariante a rotação?

Q3. O método é invariante a iluminação?

Q4. Quais as principais tendências, desafios e problemas em aberto?

O propósito das questões Q2 e Q3 é motivar a análise sobre a soluções apresentadas nos estudos primários para lidar com as duas principais interferências (iluminação e o ângulo de rotação ${ }^{1}$ ) de desempenho no reconhecimento biométrico por fusão de face e forma de andar. O objetivo da Q4 é utilizar as respostas obtidas nas questões anteriores para fazer uma conclusão do estudo realizado nessa RS.

\subsection{Protocolo da Revisão Sistemática}

De acordo com Kitchenham e Charters [16], um protocolo de RS especifica os métodos que serão utilizados para conduzi-la. Além disso, um protocolo predefinido é essencial para reduzir a possibilidade de resultados tendenciosos em pesquisa. Os autores descrevem como procurar e selecionar estudos primários relevantes, e orientam sobre a extração e análise de dados para responder a questão(ões) de pesquisa pré-definida(s). Seguindo tais diretrizes, o protoloco para a presente RS foi definido e inclui os seguintes componentes: (i) bases de dados; (ii) estratégia de pesquisa; (iii) estratégia de seleção e (iv) de avaliação de qualidade de estudos primários; (v) métodos de extração e síntese dos dados.

Seis bases de dados foram utilizadas nessa RS: Web of Science $^{2}$ (WoS), IEEE Xplore ${ }^{3}$, Science Direct ${ }^{4}$, ACM Digital Library $^{5}$, Engineering Village ${ }^{6}$ e Scopus ${ }^{7}$. Sabe-se que o motor de busca Scopus indexa todas as outras bases de dados com exceção da Web of Science, portanto os dois motores de busca usados para todas as bases de dados foram Scopus e Web of Science. Para a estratégia de pesquisa a string apresentada na Tabela 1 em sua forma básica, foi utilizada, sendo que ela foi adaptada para a sintaxe exigida em cada motor de busca. Ainda, Assumiu-se que todo estudo primário relacionado ao tema tratado nesta RS contém as palavras da string no título, no resumo ou nas palavras-chave.

\section{Tabela 1: String de busca}

TITLE-ABS-KEY ( ( face OR facial ) AND ( gait OR walking OR marching OR running ) ) AND TITLE-ABS-KEY ( statistic* OR biometr* OR identif* OR recogni* OR identif* OR authenti*))

Um conjunto de critérios de inclusão (CI) e exclusão (CE) foi especificado para selecionar os estudos primários, de forma que os estudos incluídos possuíssem um nível mínimo de qualidade, fossem primários, acessíveis e pertencentes ao escopo da RS. Os critérios aplicados nessa RS foram:

CI-1. Estudos contendo as palavras-chaves face, forma de andar, biometria ou reconhecimento, ou qualquer um

${ }^{1}$ Essas interferências são empiricamente conhecidas na área como sendo as mais influentes no reconhecimento biométrico em questão.

${ }^{2}$ http://apps.webofknowledge.com

${ }^{3}$ http://ieeexplore.ieee.org

${ }^{4}$ http://www.sciencedirect.com

${ }^{5}$ http://dl.acm.org

${ }^{6} \mathrm{http}: / /$ www.engineeringvillage.com

${ }^{7}$ https://www.scopus.com de seus sinônimos, (simultaneamente) no título, no resumo ou nas palavras chaves.

CI-2. Estudos publicados em workshop, conferência ou periódico fortemente relacionado ao escopo dessa SR.

CE-1. Estudos não disponível integralmente na Web, ou disponíveis na Web porém não acessíveis de forma gratuita via a instituição dos autores da RS.

CE-2. Estudos não revisados por pares, tais como relatórios técnicos, capítulos de livros, etc.

CE-3. Estudos não dedicados exclusivamente ao problema de fusão (ou integração) das modalidades biométricas face e forma de andar. Por exemplo, o estudo aborda as duas modalidades separadamente ou acrescenta outras modalidades como orelhas e íris.

CE-4. Estudos incompletos ou mal relatado a ponto de não ser possível identificar como os experimentos foram realizados.

CE-5. Estudos secundários ou terciários.

CE-6. Estudos classificados em áreas muito distantes de ciência da computação ou engenharia. Por exemplo, o estudo pertence à área de medicina, ciências sociais, etc.

CE-7. Estudos não inteiramente escritos na língua inglesa.

Para avaliação de qualidade dos estudos, foi estabelecido um conjunto de critérios (CQ) que permitiram analisar, medir e comparar a qualidade e a relevância dos estudos primários selecionados. O CQ foi construído para avaliar a qualidade das soluções apresentadas nos estudos primários para o problema de reconhecimento biométrico pela fusão de face e forma de andar levando em conta: o poder de generalização (A1, A4), a validação (interna e externa) (A3, A5) e a metodologia dos experimentos (A2). Também foi estabelecido um sistema de pontuação de forma que os estudos pudessem ser classificados. O CQ bem como o sistema de pontuação são apresentados na sequência.

A1. Qual o tamanho da base dados usada nos experimentos em número de indivíduos?

C1. Menor ou igual a 10: escore $=0$;

C2. Maior que 10, menor ou igual a 20: escore $=0,25$;

C3. Maior que 20, menor ou igual a 30: escore $=0,50$;

C4. Maior que 30, menor ou igual a 40: escore $=0,75$;

C5. Maior que 40: escore $=1$;

A2. Empregou validação cruzada? Não: escore $=0,5 ;$ Sim: escore $=1$;

A3. Existem grupos de controle? Por exemplo, experimentos tratando as modalidades separadamente. Não: escore $=0 ;$ Sim: escore $=1 ;$

A4. Capacidade de generalização da técnica, medida na presença de um conjunto fatores causadores de variações intraclasses:

D1. Alteração de ângulos: escore $=1 / 7$;

D2. Alteração de calçados: escore $=1 / 7$;

D3. Alteração de superfície: escore $=1 / 7$;

D4. Alteração de velocidade: escore $=1 / 7$;

D5. Carregando bagagens: escore $=1 / 7$;

D6. Alteração de roupas: escore $=1 / 7$;

D7. Alteração de tempo: escore $=1 / 7$;

A5. Comparou com os resultados da literatura? Não: escore $=0 ;$ Sim: escore $=1$;

Finalmente, para a extração e síntese dos dados os estudos foram lidos na íntegra a fim de extrair os dados relevantes 
para responder as questões de pesquisa. Um conjunto de atributos foi utilizado para sistematizar a extração e síntese dos dados: ID, título, autor, ano de publicação, fonte de publicação, e atributos descritivos conforme exposto na tabela 2. Essa tabela ainda relaciona os atributos descritivos com as três primeiras questões de pesquisa ${ }^{8}$.

Tabela 2: Formulário de extração e síntese de dados

\begin{tabular}{cl}
\hline $\begin{array}{l}\text { Questões de } \\
\text { pesquisa }\end{array}$ & Atributos \\
\hline Q1 & $\begin{array}{l}\text { Ambiente de cenas: informações relevantes da } \\
\text { base de dados que explicam as cenas. } \\
\text { Abordagem: livre de modelo ou baseada em } \\
\text { modelo. }\end{array}$ \\
& $\begin{array}{l}\text { Forma de integração: nível de fusão ou outras. } \\
\text { Controle do ambiente: são ou não controla- } \\
\text { dos. }\end{array}$ \\
Q2 & Invariante: sim ou não \\
Q3 & Invariante: sim ou não \\
\hline
\end{tabular}

\subsection{Condução da Revisão Sistemática}

A busca foi executada no período de agosto a janeiro de 2016. Os motores de busca retornaram 1973 estudos no Scopus e 5753 estudos em WoS. Os recursos de filtros oferecidos por cada motor de busca foram utilizados para aplicar, de forma automática, parte dos critérios de exclusão. Então, restaram 651 e 574 estudos, respectivamente. A partir desse ponto os critérios de exclusão foram executados manualmente por meio da análise do título e resumo de cada estudo. Na existência de dúvida, o estudo foi analisado em sua completude. Finalmente, foram selecionados 18 estudos primários, os quais foram analisados na íntegra.

\section{RESULTADOS E DISCUSSÕES}

A partir da condução do protocolo, 18 estudos publicados em um intervalo de 15 anos foram incluídos nesta RS. $\mathrm{Na}$ Tabela 3 os estudos são listados junto aos seus anos de publicação e recebem identificadores de acordo com o tipo do veículo onde foram publicados: 2 estudos em Workshop (61,11\%); 11 estudos em Conferências (27,78\%); e 5 estudos em Periódicos (27,78\%). A Figura 1 apresenta uma série temporal com a frequência de publicações por ano (2001 a 2015). Verifica-se uma média exata de publicação de 1,2 por ano, indicando uma estabilidade do tema tratado nesta RS.

Tabela 3: Estudos relevantes selecionados nesta RS

\begin{tabular}{lllllllll}
\hline ID & Ano & Ref. & ID & Ano & Ref. & ID & Ano & Ref. \\
\hline C08 & 2001 & {$[21]$} & P04 & 2007 & {$[25]$} & C10 & 2011 & {$[12]$} \\
C11 & 2002 & {$[20]$} & P05 & 2007 & {$[17]$} & P03 & 2011 & {$[4]$} \\
C05 & 2004 & {$[15]$} & P01 & 2008 & {$[26]$} & C01 & 2012 & {$[11]$} \\
C07 & 2005 & {$[27]$} & W01 & 2008 & {$[7]$} & C06 & 2012 & {$[1]$} \\
C03 & 2006 & {$[23]$} & C04 & 2009 & {$[13]$} & C02 & 2013 & {$[9]$} \\
C09 & 2006 & {$[24]$} & W02 & 2009 & {$[18]$} & P02 & 2015 & {$[22]$} \\
\hline
\end{tabular}

As próximas subseções apresentam discussões que têm o objetivo de responder as três primeiras questões de pesquisa, e também a avaliação de qualidade dos estudos. A resposta da quarta questão é apresentada nas conclusões.

${ }^{8}$ A quarta questão foi respondida a partir das respostas das três primeiras questões e por isso não consta na tabela.

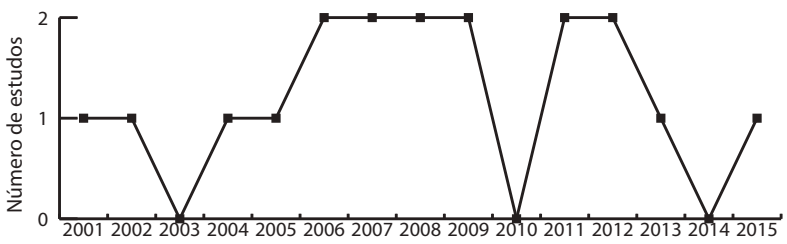

Figura 1: Número de estudos por ano de publicação

\subsection{Configurações mais exploradas (Q1)}

A resposta a essa questão pode apontar tendências e direcionar as pesquisas da área para pontos menos explorados em termos de configurações de um sistema biométrico multimodal. Cada uma das quatro especificidades desta questão é tratada em uma subseção própria.

\subsubsection{Quanto a abordagem (Q1.1)}

Dos estudos investigados $11,11 \%$ (2) apresentaram uma técnica baseada em modelo, enquanto $88,89 \%$ (16) optaram por uma técnica livre de modelo, indicando uma forte preferência em trabalhar com aparência em detrimento do trabalho com modelo geométrico. A Tabela 4 lista cada um dos estudos em relação à abordagem nele usada.

Tabela 4: Estudos por abordagem (\#: número de estudos)

\begin{tabular}{lrlr}
\hline Abordagem & ID & \% \\
\hline $\begin{array}{l}\text { Baseada } \\
\text { em modelo }\end{array}$ & 2 & C8, C11 & $11,11 \%$ \\
\hline $\begin{array}{l}\text { Livre de } \\
\text { modelo }\end{array}$ & 16 & $\begin{array}{l}\text { W01, W02, C01, C02, C03, C04, } \\
\text { C05, C06, C07, C09, C10, P01, }\end{array}$ & \\
& & P02, P03, P04, P05 & \\
\hline
\end{tabular}

As técnicas baseadas em modelo são aquelas em que os atributos são extraídos a partir de um modelo geométrico construído para representar o corpo ou a face humana, enquanto nas livres de modelo os atributos são extraídos diretamente dos pixeis das imagens. Cada abordagem possui suas vantagens e desvantagens. A primeira necessita de modelos difíceis de serem construídos e, até o momento apresenta desempenho inferior à abordagem baseada na aparência - os estudos que se baseiam em modelos apresentam, em geral, $4,7 \%$ de desempenho inferior àqueles que se baseiam na aparência; sua vantagem é que, geralmente, é invariante a escala e a rotação. Por outro lado, a abordagem livre de modelos tem a vantagem de considerar as características mais discriminativas de uma pessoa, que são aquelas que se encontram em sua aparência; no entanto, em geral, essa abordagem é sensível a quaisquer variações intraclasses, como mostrado nos experimentos dos artigos C01, C02, P02 e P05. Embora seja uma tendência a preferência em se trabalhar com a abordagem livre de modelo, a abordagem baseada em modelo precisa ser mais investigada, pois apresenta maior robustez para ambientes com usuários não cooperativos e ambientes não controlados, por ser invariante a rotação, a escala e menos sensível a ruídos [2].

\subsubsection{Quanto aos ambientes de cenas (Q1.2)}

Responder a essa questão representou uma tarefa desafiadora durante a condução dessa RS, sobretudo na etapa de extração de dados dos estudos primários. O principal 
problema encontrado foi a falta de detalhes nos relatos de alguns estudos que permitissem responder a questão e, para alguns casos, foi utilizado o processo inferência para se chegar a uma conclusão. Nesse processo, foram estabelecidas três classes de estudos: $(i)$ que possuem detalhes suficientes; $(i i)$ que não apresentam detalhes suficientes, mas fazem referência a uma base de dados pública; e (iii) que não apresentam detalhes suficientes, nem apontam referências para as bases de dados utilizadas. Para os estudos da classe (ii) o procedimento foi seguir a referência apontada para determinar as características da base de dados utilizada, assumindo que ela não foi modificada e que os pesquisadores seguiram o protocolo da base para execução dos experimentos. Já os estudos do item (iii) receberam um status de "não relatado". Tabela 5 mostra a relação completa das bases de dados utilizadas nos estudos primários.

Em relação ao ambiente de cenas, observou-se que: em 6 estudos $(33,33 \%)$ os experimentos foram conduzidos em ambientes com cenas externas; em 5 estudos $(27.78 \%)$ os experimentos foram conduzidos com cenas internas; em 4 estudos $(22,22 \%)$ os experimentos foram conduzidos em ambientes heterogêneos com cenas internas e externas; e 3 estudos não relataram essa informação $(16,67 \%)$. Em ambientes controlados, onde existe o controle dos fatores causadores de variações intraclasses, geralmente, as cenas são internas e quanto maior o controle sobre as condições de ambiente mais se aproxima da solução trivial para o problema de reconhecimento biométrico pela fusão de face e forma de andar. Em contrapartida, em ambientes não controlados onde as cenas são externas, quanto mais fatores causadores de variações intraclasses mais difícil torna se a solução. É possível inferir a partir dos resultados apresentados nos estudos que aqueles que usam ambientes controlados apresentam um desempenho melhor(cerca de 15,4\% maior). Entretanto, há estudos em ambientes não controlado (C01 e P05) que consegue obter ótimos desempenhos (como P05 que chega a 89\%).

\subsubsection{Quanto ao esquema de integração (Q1.3)}

Apenas um estudo (C05) apresentou um relato referente a um esquema de integração diferente do esquema baseada em nível de fusão (BNF), e ainda, o mesmo estudo também investigou o BNF em nível de escores. Assim, a presente análise foca especificamente em BNF. O esquema de integração BNF foi investigado em todos os 18 estudos. Desses estudos, nenhum investigou a fusão no nível sensorial; 7 $(38,89 \%)$ estudos investigaram a fusão no nível de extração de características (C03, P01, P02, C06, P03, C08, W02); $11(61,11 \%)$ investigaram a fusão no nível de escores (W01, $\mathrm{C} 01, \mathrm{C} 02, \mathrm{C} 04, \mathrm{C} 05, \mathrm{C} 07, \mathrm{P} 04, \mathrm{C} 09, \mathrm{C} 10, \mathrm{C} 11, \mathrm{P} 05)$; e 1 $(5,56 \%)$ estudo investigou a fusão no nível de decisão (C02). Esses resultados evidenciam a preferência da comunidade de pesquisadores pelo estudo da BNF no nível de escores, seguida pela fusão no nível de extração de características.

Um destaque importante deve ser feito à ausência de estudos que tratam BNF no nível sensorial. Essa ausência faz sentido porque, até o melhor de nosso conhecimento, ainda não se conhece técnicas para fusão de face e forma de andar nesse nível, tampouco se conhece alguma possível vantagem nesse tipo de fusão para esse caso . Empiricamente, tratase de um problema em aberto confirmado pelos resultados obtidos nessa RS.

A fusão no nível de escores é o que apresenta maior custobenefício, considerando como custo os esforços necessários para a integração e como benefícios os ganhos em desempenho. Esse fato é comprovado pelos resultados e análises fornecidas nos estudos W01, C01, C02, C04, C05, C07, P04, C09, C10, C11 e P05. A fusão no nível de características possui restrições quanto à compatibilidade da dimensão dos vetores, assim a menos que seja optado por uma simples concatenação dos vetores de características como em C03, P01 e W02, realizar a fusão é um problema difícil. A fusão por concatenação é a mais simples, porém sua desvantagem é o crescimento da dimensionalidade do novo espaço de características gerado. Esse crescimento aumenta a complexidade do problema exigindo cada vez mais dados para se chegar a um modelo. No estudo W02 por exemplo, a dimensionalidade aumentou de 25 e 13 para face e forma de andar, respectivamente, para 38 no espaço sintético. A fusão no nível de decisão está sendo timidamente estudada e requer mais esforços para evidenciar suas possíveis vantagens e desvantagens.

Existe uma forte tendência para o esquema de integração BNF comparado com outros esquemas de integração descritos na literatura. A discrepância é grande considerando que em $100 \%$ dos estudos primários analisados foi investigado o sistema de integração BNF e apenas em 5,56\% dos estudos foi investigado uma integração hierárquica (um estudo investigou os dois esquemas).

\subsubsection{Quanto a alterações de ambiente (Q1.4)}

Desde que o objetivo dessa questão é analisar quais são as principais alterações de ambiente presentes nas cenas, para respondê-la considerou-se apenas estudos que apresentaram experimentos conduzidos na presença de tais alterações (veja as alterações de interesse (D1 a D7) na seção 3.3. Não necessariamente os sistemas construídos para o reconhecimento biométrico pela fusão de face e forma de andar nos estudos analisados apresentaram uma sub-rotina específica para lidar com essas variações. A tabela 6 apresenta os estudos agrupados por tipo alteração presente nos experimentos relatados. As possíveis alterações não se limitam às consideradas nessa RS.

Embora na teoria exista ambiente controlado, na prática não existe a ideia de ambiente totalmente controlado. Em todos os estudos, os experimentos foram conduzidos na presença de algum tipo de alteração. Por exemplo, embora houvesse a tentativa em excluir as alterações de ângulos (D1) nos estudos C02, C03, P01, C07, P04 e C09, os experimentos apresentaram algum tipo de alteração dentre as citadas nessa questão ou não limitada a elas.

\subsection{O método é invariante a rotação? $\mathrm{E}$ ilumi- nação? (Q2 e Q3)}

Essas duas questões têm o objetivo de esclarecer se os sistemas construídas para o problema de reconhecimento biométrico pela fusão de face e forma de andar aplicam técnicas específicas para lidar com o problema de iluminação e ângulo de rotação. Cinco estudos primários (27,78\%) aplicaram alguma técnica para tratar o problema do ângulo de visualização da câmera, e nenhum estudo tratou o problema de variação de iluminação. A tabela 7 relaciona os estudos de acordo com a apresentação de técnicas para tratamento desses dois problemas.

O problema de reconhecimento biométrico pela fusão de face e forma é desafiador, pois se por um lado a fusão é responsável por amenizar os efeitos das alterações com um 
Tabela 5: Bases de dados utilizadas nos estudos

\begin{tabular}{|c|c|c|c|c|c|c|}
\hline Base de dados & $\begin{array}{l}\text { \# de in- } \\
\text { divíduos }\end{array}$ & $\begin{array}{l}\text { \# de se- } \\
\text { quências }\end{array}$ & Ano & Ambiente & Variações & ID \\
\hline CASIA dataset B & 124 & 13640 & 2005 & indoor & 11 ângulos, roupas, carregando objetos & C06,W02 \\
\hline CASIA d.B+ORL & 40 & 400 & $1992 / 2005$ & 5 indoor & 11 ângulos, roupas, carregando objeto, tempo & P02 \\
\hline CASIA dataset A & 20 & 240 & 2001 & outdoor & 3 ângulos, iluminação & W01 \\
\hline HI. Gait Challenge & 122 & 1870 & 2001 & heterogênio & $\begin{array}{l}2 \text { ângulos, superfície, calçados, carregando ob- } \\
\text { jetos, tempo }\end{array}$ & $\mathrm{C} 01, \mathrm{P} 05$ \\
\hline $\mathrm{KTH}$ & 25 & 100 & 2004 & heterogênio & tempo, roupas & $\mathrm{P} 03, \mathrm{C} 10$ \\
\hline NIST database & 30 & NR & NR & outdoor & ângulos, iluminação & $\mathrm{C} 05$ \\
\hline TUM-GAID & 305 & 3370 & 2012 & indoor & mochila, sapato, agasalho e tempo & $\mathrm{C} 02$ \\
\hline Própria & 31 & 186 & 2009 & NR & nehuma & $\mathrm{C} 04$ \\
\hline Própria & 45 & 100 & 2006 & indoor & tempo, roupas, Iluminação & P01 \\
\hline Própria & 45 & 90 & 2005 & outdoor & roupas, tempo, iluminação & P04 \\
\hline Própria & 46 & 92 & 2005 & outdoor & roupas, tempo, iluminação & $\mathrm{C} 09$ \\
\hline Própria & 14 & 28 & NR & outdoor & tempo e roupas & $\mathrm{C} 07$ \\
\hline Própria & 12 & 54 & NR & NR & ângulos & $\mathrm{C} 08$ \\
\hline Própria & 22 & 206 & NR & $\mathrm{NR}$ & tempo e ângulos & $\mathrm{C} 11$ \\
\hline Não identificada & 46 & 92 & 2006 & outdoor & iluminação & $\mathrm{C} 03$ \\
\hline
\end{tabular}

Tabela 6: Estudos por tipos de alteração (\#: número de estudos)

\begin{tabular}{|c|c|c|c|}
\hline AL & \# & ES & $\%$ \\
\hline D1 & 12 & $\begin{array}{l}\text { W01, C01, C04, C05, P02, C06, P03, } \\
\text { C08, W02, C10, C11, P05 }\end{array}$ & $66,67 \%$ \\
\hline D2 & 7 & $\mathrm{C} 01, \mathrm{C} 02, \mathrm{P} 03, \mathrm{P} 04, \mathrm{C} 09, \mathrm{C} 10, \mathrm{P} 05$ & $38,89 \%$ \\
\hline D3 & 2 & $\mathrm{C} 01, \mathrm{P} 05$ & $11,11 \%$ \\
\hline D4 & 2 & $\mathrm{C} 01, \mathrm{P} 05$ & $11,11 \%$ \\
\hline D5 & 5 & $\mathrm{C} 01, \mathrm{C} 02, \mathrm{C} 06, \mathrm{~W} 02, \mathrm{P} 05$ & $27.78 \%$ \\
\hline D6 & 12 & $\begin{array}{l}\mathrm{C} 01, \mathrm{C} 02, \mathrm{P} 01, \mathrm{C} 04, \mathrm{P} 02, \mathrm{C} 07, \mathrm{P} 03, \\
\mathrm{P} 04, \mathrm{C} 09, \mathrm{C} 10, \mathrm{C} 11, \mathrm{P} 05\end{array}$ & $66,67 \%$ \\
\hline D7 & 12 & $\begin{array}{l}\mathrm{C} 01, \mathrm{C} 02, \mathrm{P} 01, \mathrm{P} 02, \mathrm{C} 06, \mathrm{C} 07, \mathrm{P} 03 \text {, } \\
\mathrm{P} 04, \mathrm{C} 09, \mathrm{~W} 02, \mathrm{C} 10, \mathrm{P} 05\end{array}$ & $66,67 \%$ \\
\hline
\end{tabular}

rápido ganho no desempenho do sistema, por outro o sistema precisa lidar com os problemas deixados por ambas a biometrias. Embora na área de reconhecimento de face existam técnicas eficientes para lidar com o problema da iluminação, na área de integração da face com forma de andar ainda não há na literatura, de acordo com os resultados dessa RS, uma iniciativa que adiciona uma sub-rotina específica para esse tipo de alteração.

Tabela 7: Estudos agrupados como variante ou invariante a rotação ou a iluminação

\begin{tabular}{|c|c|c|}
\hline & Rotação & Iluminação \\
\hline Invariante & $\begin{array}{l}\text { W01, C05, C08, } \\
\text { W02, C11 }\end{array}$ & \\
\hline Variante & $\begin{array}{l}\text { C01, C02, C03, P01, } \\
\text { C04, P02, C06, C07, } \\
\text { P03, P04, C09, C10, } \\
\text { P05 }\end{array}$ & $\begin{array}{l}\text { W01, C01, C02, C03, } \\
\text { P01, C04, C05, P02, } \\
\text { C06, C07, P03, C08, } \\
\text { P04, C09, W02, C10, } \\
\text { C11, P05 }\end{array}$ \\
\hline
\end{tabular}

\subsection{Avaliação de qualidade}

O instrumento de avaliação de qualidade (seção 3.3 possibilitou a geração um escore para cada estudo primário. $\mathrm{O}$ primeiro passo nesse processo foi somar a pontuação obtida para cada questão do conjunto de critérios CQ. Em seguida, foi aplicada a normalização Min-Max para restringir a soma ao intervalo $[0,1]$. A tabela 8 mostra o resultado da avaliação de qualidade dos estudos, e a figura 2 mostra a pontuação obtida (o escore) por cada estudo primário. A médias dos escores normalizados é 0,54 . Observando a figura 2 observase que somente os estudos C01, C02, C06, P05, W02 e P02 possuem qualidade acima da média. O estudo que obteve o melhor escore foi publicado em uma conferência.

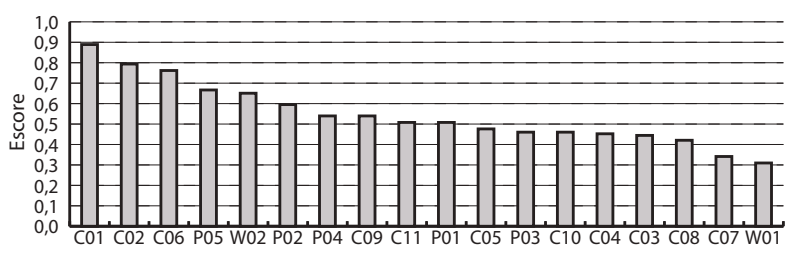

Figura 2: Qualidade (escore) de cada estudo

O instrumento de avaliação da qualidade foi projetado para considerar a metodologia dos experimentos (critérios A1, A2 e A3), o poder de generalização dos sistemas (critério A4), e a comparação com resultados da literatura (critério A5). Considerando a metodologia dos experimentos, foram avaliadas a quantidade de indivíduos presente na base de dados (A1), se houve validação cruzada (A2), e se foram usados grupos de controle para comparação de resultados (A3). Algumas características desse processo de avaliação merecem destaque: somente os estudos W02, C05, C08, C11 e P02 apresentaram validação cruzada, uma metodologia de aferição de robustez fortemente usada pela comunidade científica de reconhecimento de padrões; quanto maior a quantidade de alterações consideradas nos experimentos melhor é o escore do estudo em relação ao poder de generalização do sistema; o uso de bases de dados próprias impossibilita a comparação de resultados obtidos nos estudos com outros presentes na literatura da área, e esse fato pesou negativamente nos escores dos estudos C01, P05, W02, P02, P04, C09, P01, C05, P03, C10, C04, C03, C08, C07 e W01.

A robustez dos sistemas apresentados nos estudos analisados, em termos de acurácia nos experimentos, não pode ser comparada devido ao fato que as condições de ambiente 
Tabela 8: Avaliação de qualidade dos estudos primários por meio da aplicação do conjuntos de critérios CQ

\begin{tabular}{|c|c|c|c|c|c|c|c|c|c|c|c|c|c|c|c|c|c|c|}
\hline Critérios & & & & & & & & & Est & dos & & & & & & & & \\
\hline $\begin{array}{r}\text { ID } \\
\mathrm{A} 1 . \mathrm{C} 1\end{array}$ & W01 & $\mathrm{C} 01$ & $\mathrm{C} 02$ & $\mathrm{C} 03$ & P01 & $\mathrm{C} 04$ & $\mathrm{C} 05$ & P02 & $\mathrm{C} 06$ & $\mathrm{C} 07$ & P03 & $\mathrm{C} 08$ & P04 & $\mathrm{C} 09$ & W02 & $\mathrm{C} 10$ & C11 & P05 \\
\hline A1.C2 & $\mathrm{x}$ & & & & & & & & & $\mathrm{x}$ & & $\mathrm{x}$ & & & & & & \\
\hline A1.C3 & & & & & & & $\mathrm{x}$ & & & & $\mathrm{x}$ & & & & & $\mathrm{x}$ & $\mathrm{x}$ & \\
\hline A1.C4 & & & & & & $\mathrm{x}$ & & $\mathrm{x}$ & & & & & & & & & & \\
\hline A1.C5 & & $\mathrm{x}$ & $\mathrm{x}$ & $\mathrm{x}$ & $\mathrm{x}$ & & & & $\mathrm{x}$ & & & & $\mathrm{x}$ & $\mathrm{x}$ & $\mathrm{x}$ & & & $\mathrm{x}$ \\
\hline A2.S & & & & & & & $\mathrm{x}$ & $\mathrm{x}$ & & & & $\mathrm{x}$ & & & $\mathrm{x}$ & & $\mathrm{x}$ & \\
\hline A2.N & $\mathrm{x}$ & $\mathrm{x}$ & $\mathrm{x}$ & $\mathrm{x}$ & $\mathrm{x}$ & $\mathrm{x}$ & & & $\mathrm{x}$ & $\mathrm{x}$ & $\mathrm{x}$ & & $\mathrm{x}$ & $\mathrm{x}$ & & $\mathrm{x}$ & & $\mathrm{x}$ \\
\hline $\begin{array}{r}\text { A3.S } \\
\text { A3.N }\end{array}$ & $\mathrm{x}$ & $\mathrm{x}$ & $\mathrm{x}$ & $\mathrm{x}$ & $\mathrm{x}$ & $\mathrm{x}$ & $\mathrm{x}$ & $\mathrm{x}$ & $\mathrm{x}$ & $\mathrm{x}$ & $\mathrm{x}$ & $\mathrm{x}$ & $\mathrm{x}$ & $\mathrm{x}$ & $\mathrm{x}$ & $\mathrm{x}$ & $\mathrm{x}$ & $\mathrm{x}$ \\
\hline A4.D1 & $\mathrm{x}$ & $\mathrm{x}$ & & & & $\mathrm{x}$ & $\mathrm{x}$ & $\mathrm{x}$ & $\mathrm{x}$ & & $\mathrm{x}$ & $\mathrm{x}$ & & & $\mathrm{x}$ & $\mathrm{x}$ & $\mathrm{x}$ & $\mathrm{x}$ \\
\hline A4.D2 & & $\mathrm{x}$ & $\mathrm{x}$ & & & & & & & & $\mathrm{x}$ & & $\mathrm{x}$ & $\mathrm{x}$ & & $\mathrm{x}$ & & $\mathrm{x}$ \\
\hline A4.D3 & & $\mathrm{x}$ & & & & & & & & & & & & & & & & $\mathrm{x}$ \\
\hline A4.D4 & & $\mathrm{x}$ & & & & & & & & & & & & & & & & $\mathrm{x}$ \\
\hline A4.D5 & & $\mathrm{x}$ & $\mathrm{x}$ & & & & & & $\mathrm{x}$ & & & & & & $\mathrm{x}$ & & & $\mathrm{x}$ \\
\hline A4.D6 & & $\mathrm{x}$ & $\mathrm{x}$ & & $\mathrm{x}$ & $\mathrm{x}$ & & $\mathrm{x}$ & & $\mathrm{x}$ & $\mathrm{x}$ & & $\mathrm{x}$ & $\mathrm{x}$ & & $\mathrm{x}$ & $\mathrm{x}$ & $\mathrm{x}$ \\
\hline A4.D7 & & $\mathrm{x}$ & $\mathrm{x}$ & & $\mathrm{x}$ & & & $\mathrm{x}$ & $\mathrm{x}$ & $\mathrm{x}$ & $\mathrm{x}$ & & $\mathrm{x}$ & $\mathrm{x}$ & $\mathrm{x}$ & $\mathrm{x}$ & & $\mathrm{x}$ \\
\hline A5.S & & $\mathrm{x}$ & $\mathrm{x}$ & & & & & & $\mathrm{x}$ & & & & & & & & & \\
\hline A6.N & $\mathrm{x}$ & & & $\mathrm{x}$ & $\mathrm{x}$ & $\mathrm{x}$ & $\mathrm{x}$ & $\mathrm{x}$ & & $\mathrm{x}$ & $\mathrm{x}$ & $\mathrm{x}$ & $\mathrm{x}$ & $\mathrm{x}$ & $\mathrm{x}$ & $\mathrm{x}$ & $\mathrm{x}$ & $\mathrm{x}$ \\
\hline Soma & 1.89 & 4.50 & 4.07 & 2.50 & 2.79 & 2.54 & 2.64 & 3.18 & 3.93 & 2.04 & 2.57 & 2.39 & 2.93 & 2.93 & 3.43 & 2.57 & 2.79 & 3.50 \\
\hline Escore & 0.31 & 0.89 & 0.79 & 0.44 & 0.51 & 0.45 & 0.48 & 0.60 & 0.76 & 0.34 & 0.46 & 0.42 & 0.54 & 0.54 & 0.65 & 0.46 & 0.51 & 0.67 \\
\hline
\end{tabular}

não são as mesmas em todos eles. Contudo é possível observar que estudos onde os experimentos foram conduzidos em bases próprias, em geral, apresentam, em média, acurácia muito superior aos estudos com experimentos em bases públicas. Nesse caso, foi considerado que esses resultados possuem baixa validade externa. Os estudos P01, P03 e P04 ficaram com escores abaixo da média: P01 e P04 utilizaram bases de dados próprias ( tabela 5), P03 utilizou uma base de dados pública porém alterada (impossibilitando comparação com resultados da literatura). O estudo C01 que obteve o maior escore utilizou a base de dados mais desafiadora conhecida atualmente: Human ID Gait Challenge, que possui 12 conjuntos de testes, contendo 32 tipos de alteração ao todo. Dessa forma, foi possível contemplar todas os 7 tipos de alterações consideradas no critério A4. Um fator determinante nos três melhores estudos, de acordo com o instrumento de avaliação de qualidade, é o fato deles terem comparado seus resultados com os resultados da literatura.

\section{AMEAÇAS À VALIDADE}

Nesse trabalho foram consideradas a validade interna e a validade externa. Diz-se que uma pesquisa possui alta validade interna quando se tem certeza que suas hipóteses apresentam uma única explanação plausível para os dados. Nesse sentido, as ameaças à validade interna dessa RS são algumas decisões subjetivas tomadas durante a extração de dados. Alguns estudos primários não forneceram um relato claro sobre metodologia e resultados, com detalhes suficientes para a aplicação objetiva dos critérios de inclusão e exclusão e do procedimento de extração de dados. Então, foi aplicada a estratégia de teste-reteste para minimizar as ameaças a validade interna dessa revisão. Nessa estratégia a extração de dados foi aplicada duas vezes. Se não houver divergência, as informações são ditas confiáveis.

A validade externa refere-se a capacidade de generalização de resultados e conclusões. Nesse sentido os resultados dessa revisão não podem generalizar para uma seleção de estudos primária mais ampla, indo além das bases de dados utilizadas. Os resultados foram obtidos a partir da execução de procedimentos seguindo uma metodologia de modo que qualquer pesquisador possa reproduzi-los. No entanto, a extração de dados é uma fonte de ameaça, uma vez que existem fatores subjetivos inerentes ao avaliador. Outra ameaça foi a resposta a Q1.2 conforme explicado na seção 4.1.2.

\section{CONCLUSÃO}

Essa RS identificou, avaliou e interpretou 18 estudos primários relacionados ao problema de fusão de face e forma de andar para o reconhecimento biométrico. As três primeiras questões de pesquisa foram respondidas e a partir de tais respostas, a questão Q4 (tendências, desafios e problemas em aberto) pode ser discutida para concluir esse artigo. A partir dos resultados já contabilizados é possível afirmar que abordagem livre de modelo tem prevalência sobre a abordagem baseada em modelo, e essa é a primeira tendência encontrada. A abordagem baseada em modelo precisa ser mais estudada a fim de aumentar sua eficiência visto que apresenta maior robustez para ambiente não controlado pelo fato de ser invariante a rotação e a escala e não ser sensível iluminação e outros fatores. Quanto ao ambiente de captura das cenas, constatou-se que a maior quantidade estudos se destinou a trabalhar com o ambiente externo, sendo essa a segunda tendência encontrada. A terceira tendência diz respeito ao esquema de integração BNF nível de escores.

Nenhum estudo tratou a fusão no nível sensorial e esse é um problema em aberto: encontrar técnicas que lidem com a fusão no nível sensorial para descobrir suas vantagens e desvantagens. Analisando os resultados da Q1.3, 66,67\% dos estudos primários apresentam uma fusão pós-classificação (ou pós-mapeamento). Quanto aos resultados obtidos para as questões Q2 e Q3, existe uma lacuna no tratamento de alterações de iluminação nos sistemas biométricos com fusão de face e forma de andar. Embora o reconhecimento de forma de andar sofra pouco com essa alteração (somente em casos extremos no qual a sombra projetada pela iluminação interfere na detecção da silhueta [3]), o problema de iluminação interfere no reconhecimento de face [2].

Fusão de face e forma de andar apresenta ganho no desem- 
penho geral do sistema, no entanto, soma uma quantidade maior de fatores causadores de variações intraclasses, tornando mais difícil o tratamento de todos esses fatores em um único modelo de reconhecimento biométrico. De acordo com as constatações dessa RS, a taxa de produção científica tratando o problema de fusão de face e forma de andar para o reconhecimento biométrico está estabilizada em 1,2 estudos por ano e algumas tendências de pesquisa já são observadas. No entanto, trata-se de uma taxa baixa evidenciando que a área ainda se encontra em uma fase de desenvolvimento rudimentar e essa RS contribui com o desenvolvimento da área ao apontar, a partir de um processo sistemático de análise da área, as lacunas que precisam ser investigadas.

\section{REFERÊNCIAS}

[1] M. S. Almohammad, G. I. Salama, and T. A. Mahmoud. Human identification system based on feature level fusion using face and gait biometrics. In Proc of Int. Conf. on Engineering and Technology (ICET), pages 1-5, Oct. 2012.

[2] I. Buciu. Challenges and specifications for robust face and gait recognition systems for surveillance application. J. of Electrical and Electronics Engineering, 7(1):25-30, 2014.

[3] R. Chellappa and G. Aggarwal. Advances in Biometrics: Sensors, Algorithms and Systems, chapter Pose and Illumination Issues in Face- and Gait- Based Identification, pages 307-322. Springer London, London, 2008.

[4] G. Chetty and M. Singh. Information fusion for identity verification. World Academy of Science, Engineering and Technology, 5(5):979-984, 2011.

[5] M. Eskandari, Ö. Toygar, and H. Demirel. Feature extractor selection for face-iris multimodal recognition. Signal, Image and Video Processing, 8(6):1189-1198, 2014.

[6] M. Gavrilova, M. Monwar, and I. Global. Multimodal Biometrics and Intelligent Image Processing for Security Systems. IGI Global, 2013.

[7] X. Geng, L. Wang, M. Li, Q. Wu, and K. Smith-Miles. Adaptive fusion of gait and face for human identification in video. In Proc. of IEEE Workshop on App. of Comp. Vision (WACV), pages 1-6, Jan. 2008.

[8] Y. Guan, X. Wei, C.-T. Li, and Y. Keller. People identification and tracking through fusion of facial and gait features. In V. Cantoni, D. Dimov, and M. Tistarelli, editors, Biometric Authentication, pages 209-221. Springer, 2014.

[9] Y. Guan, X. Wei, C.-T. Li, G. Marcialis, F. Roli, and M. Tistarelli. Combining gait and face for tackling the elapsed time challenges. In Proc. of IEEE 6th Int. Conf. on Biometrics: Theory, App. and Sys. (BTAS), pages 1-8, Sept 2013.

[10] F. He, Y. Liu, X. Zhu, C. Huang, Y. Han, and Y. Chen. Score level fusion scheme based on adaptive local gabor features for face-iris-fingerprint multimodal biometric. J. of Electronic Imaging, 23(3):033019, 2014.

[11] M. Hofmann, S. Schmidt, A. Rajagopalan, and G. Rigoll. Combined face and gait recognition using alpha matte preprocessing. In Proc. of 5th IAPR Int. Conf. on Biometrics (ICB), pages 390-395, Mar. 2012.
[12] E. Hossain and G. Chetty. Multimodal face-gait fusion for biometric person authentication. In Proc. of 9th IFIP Int. Conf. on Embedded and Ubiquitous Computing (EUC), pages 332-337, Oct. 2011.

[13] X. Hou and Z. Liu. Fusion of face and gait for human recognition in video sequences. In Proc. of Int. Conf. on Inf. Technology and Comp. Science (ITCS), volume 1, pages 577-580, Jul. 2009.

[14] A. Jain, A. Ross, and S. Prabhakar. An introduction to biometric recognition. Circuits and Sys. for Video Tech., IEEE Transactions on, 14(1):4-20, Jan 2004.

[15] A. Kale, A. Roychowdhury, and R. Chellappa. Fusion of gait and face for human identification. In Proc. of IEEE Int. Conf. on Acoustics, Speech, and Signal Processing (ICASSP), volume 5, pages V-901-4, May 2004.

[16] B. Kitchenham and S. Charters. Guidelines for performing systematic literature reviews in software engineering, 2007.

[17] Z. Liu and S. Sarkar. Outdoor recognition at a distance by fusing gait and face. Image and Vision Computing, 25(6):817-832, 2007.

[18] L. Qi-Shen, L. Zhi-Tian, and Z. Dan-dan. Integration of gait and side face for human recognition in video. In Proc. of 2nd Int. Symp. on Electronic Commerce and Security (ISECS), volume 2, pages 65-69, May 2009.

[19] A. Ross and A. Jain. Information fusion in biometrics. Pattern Recognition Letters, 24(13):2115-2125, 2003.

[20] G. Shakhnarovich and T. Darrell. On probabilistic combination of face and gait cues for identification. In Proc. of 5th IEEE Int. Conf. on Automatic Face and Gesture Recognition, pages 169-174, May 2002.

[21] G. Shakhnarovich, L. Lee, and T. Darrell. Integrated face and gait recognition from multiple views. In Proc. of IEEE Conf. on Comp. Vision and Pattern Recognition (CVPR), volume 1, pages I-439-I-446, 2001.

[22] X. Xing, K. Wang, and Z. Lv. Fusion of gait and facial features using coupled projections for people identification at a distance. IEEE Signal Processing Letters, 22(12):2349-2353, Dec. 2015.

[23] X. Zhou and B. Bhanu. Feature fusion of face and gait for human recognition at a distance in video. In Proc. of 18th Int. Conf. on Pattern Recognition (ICPR), volume 4, pages 529-532, 2006.

[24] X. Zhou and B. Bhanu. Integrating face and gait for human recognition. In Proc. of Conf. on Comp. Vision and Pattern Recognition Workshop (CVPRW), pages 55-55, Jun. 2006.

[25] X. Zhou and B. Bhanu. Integrating face and gait for human recognition at a distance in video. IEEE Trans. on Sys., Man, and Cyber., Part B: Cyber., 37(5):1119-1137, Oct. 2007.

[26] X. Zhou and B. Bhanu. Feature fusion of side face and gait for video-based human identification. Pattern Recognition, 41(3):778-795, 2008.

[27] X. Zhou, B. Bhanu, and J. Han. Human recognition at a distance in video by integrating face profile and gait. In T. Kanade, A. Jain, and N. Ratha, editors, Audioand Video-Based Biometric Person Authentication, volume 3546 of LNCS, pages 533-543. Springer, 2005. 